\title{
Stationary and pulsating dissipative light bullets from a collective variable approach
}

\author{
A. Kamagate, Ph. Grelu, and P. Tchofo-Dinda \\ Institut Carnot de Bourgogne, UMR 5209 CNRS, Université de Bourgogne, 9 Avenue Savary, BP 47870, 21078 Dijon Cedex, France
}

J. M. Soto-Crespo

Instituto de Óptica, C.S.I.C., Serrano 121, 28006 Madrid, Spain

\author{
N. Akhmediev \\ Optical Sciences Group, Research School of Physical Sciences and Engineering, The Australian National University, \\ Canberra ACT 0200, Australia \\ (Received 11 April 2008; published 23 February 2009)
}

\begin{abstract}
A collective variable approach is used to map domains of existence for $(3+1)$-dimensional spatiotemporal soliton solutions of a complex cubic-quintic Ginzburg-Landau equation. A rich variety of evolution behaviors, which include stationary and pulsating dissipative soliton dynamics, is revealed. Comparisons between the results obtained by the semianalytical approach of collective variables, and those obtained by a purely numerical approach show good agreement for a wide range of equation parameters. This also demonstrates the relevance of the semianalytical method for a systematic search of stability domains for spatiotemporal solitons, leading to a dramatic reduction of the computation time.
\end{abstract}

DOI: 10.1103/PhysRevE.79.026609

PACS number(s): 05.45.Yv, 42.65.Tg, 42.65.Sf, 47.20.Ky

\section{INTRODUCTION}

In physical systems that exchange energy with the environment, an efficient use of nonlinear dissipation allows the formation of stable localized structures [1]. During the past few years, numerous nonlinear open systems have been studied in the frame of "dissipative soliton" theory, a fast emerging field of nonlinear science [2,3]. In general, dissipative solitons are fixed localized solutions that result from a double balance: between dispersive and nonlinear conservative effects on the one hand and between gain and loss on the other hand. These two balances are strongly coupled, providing unique properties to these localized formations. Thus, dissipative solitons are not merely extensions of the solitons that have been studied for a long time in Hamiltonian systems. They are usually attractors in the infinite-dimensional dynamical systems under consideration. As a result, soliton solutions are stable with respect to parameter fluctuations and noise. Dissipative solitons are also tolerant to the inclusion of additional higher-order terms into the master equation or to the increase of the spatiotemporal dimensionality of the problem.

Dissipative systems in nonlinear optics admit stable solitons in one, two, and three dimensions [4,5]. The stability of localized optical structures in more than one dimension is a nontrivial issue even for conservative systems. Generally, one has to deal with marginal stability and collapse [6,7]. In the latter case, the nonlinear medium is assumed to be homogeneous, which excludes field localization in waveguides and cavities. Instead, the localized optical field creates its own waveguide in the nonlinear medium and sustains its localization during propagation.

Master equations for wave propagation in dissipative nonlinear media can be based on a cubic-quintic complex Ginzburg-Landau equation (CGLE) $[8,9]$. The CGLE has been widely used in nonlinear dissipative optics, due to the clear physical meaning of all its terms in any particular ap- plication. Among the important applications are passively mode-locked laser systems and optical transmission lines. Exact analytical soliton solutions have been derived in the case of the one-dimensional CGLE, although all of them have proved to be unstable. However, stable solutions were found numerically in specific domains of the system parameters [8]. In addition to stationary solitons, there are pulsating ones, which also exist in separate regions of the parameter space. For one- and two-dimensional solitons, their regions of existence have been studied extensively, either numerically [10] or with the semianalytical "method of moments" $[11,12]$.

Extrapolating the methodology and results from the (1 $+1)$-dimensional $[(1+1) \mathrm{D}]$ evolution problem, we can expect that in higher dimensions the only way of finding localized structures is an entirely numerical procedure. Some of the $(3+1) \mathrm{D}$ soliton solutions admit spherical symmetry between temporal and all spatial variables. Thus, if we restrict ourselves to stationary spherically symmetric solutions, it is possible to reduce the master equation to an ordinary differential equation (ODE), which can be solved numerically with relative ease [4,13]. However, when modeling realistic physical systems, it is important to decouple temporal and spatial effects. For instance, propagation can take place also in media with normal dispersion. Besides, temporal bandwidth limitation, or spectral filtering, can largely dominate spatial filtering effects. These effects will break the spherical symmetry. Solutions without spherical symmetry may exist for many other reasons. Recently, the existence of stable light bullets with complicated shapes was demonstrated using the 3D CGLE for any sign of chromatic dispersion $[5,14]$ and for a wide range of equation parameters. Stable vortex toroidal spatiotemporal solitons were also found within the same CGLE model $[15,16]$. Even the ability of light bullets to form complexes was unveiled from numerical simulations $[17,18]$. 
Solving numerically a $(3+1) \mathrm{D}$ equation for a given set of parameters and a given initial condition is an extremely lengthy and costly procedure, which can take up to several days in a standard PC [14]. A task that is even more tedious is the mapping between the type of solution and the set of parameters of the equation. Typically, we deal with a 5D space of parameters. In this context, it is important to develop theoretical tools that can perceive soliton solutions more efficiently and envisage their domains of existence. A few approximate, semianalytical methods based on various physical backgrounds were developed and applied to study nonlinear pulse propagation. Several of them make use of a trial function and its associated finite-dimensional dynamical system. Reductions to a finite-dimensional dynamical system use variational principles $[19,20]$, the method of moments $[11,12,21]$, and a collective variable approach [22]. The resulting dynamical system controls the evolution of a finite number of parameters such as the pulse width, chirp, amplitude, etc. Dealing with a finite number of variables reduces significantly the computation time, but at the expense of accuracy.

Dealing with more than three parameters in the trial function usually causes significant difficulties. In this respect, $(3+1)$ D problems comprise a special class because the number of parameters in the trial function has to be at least 5 . Reduction to three parameters becomes possible when we restrict the trial function to be spherically symmetric. This limitation is too strong and allows us to get a very limited view of how optical bullets may evolve. Thus, lifting these restrictions is essential for further progress. Recently, the Lagrangian approach was applied to a $(3+1) \mathrm{D}$ CGLE with spherical symmetry, using a Gaussian trial function with four [19] or eight parameters [23]. In the present work, we show that the approach of "collective variables" is a clearly formulated technique that is particularly well suited for finding the approximate localized solutions of the $(3+1)$ D CGLE. It does not require spherical symmetry of the CGLE and is compatible with a number of trial functions. Using this technique, we here find a rich variety of solutions that include stationary, pulsating, and a new type of breathing solutions. Another advantage of the technique is the dramatic reduction of the computer time that is required to find approximate location of specific solutions in the parameter space of the CGLE.

The remainder of the paper is organized as follows: in Sec. II we describe the collective variable approach and our procedure of determination of the stability domains of the light bullets. In Sec. III we present the stability domain of stationary light bullets, as well as a comparison with exact numerical calculations. Section IV is devoted to the findings of pulsating light bullets. Finally, in Sec. V we give some concluding remarks.

\section{COLLECTIVE VARIABLE APPROACH}

\section{A. Ginzburg-Landau equation model}

We consider the propagation of light bullets in a system described by an extended complex cubic-quintic GinzburgLandau equation model. This model includes cubic and quin- tic nonlinearities of dispersive and dissipative types, and we added transverse operators to take into account spatial diffraction in the paraxial wave approximation. The normalized propagation equation reads

$$
\begin{aligned}
i \psi_{z} & +\frac{D}{2} \psi_{t t}+\frac{1}{2} \psi_{x x}+\frac{1}{2} \psi_{y y}+|\psi|^{2} \psi+\nu|\psi|^{4} \psi \\
& =i \delta \psi+i \epsilon|\psi|^{2} \psi+i \beta \psi_{t t}+i \mu|\psi|^{4} \psi .
\end{aligned}
$$

The optical envelope $\psi$ is a complex function of four real variables $\psi=\psi(x, y, t, z)$, where $t$ is the retarded time in the frame moving with the pulse, $z$ is the propagation distance, and $x$ and $y$ are the two transverse coordinates. Equation (1) is written in normalized form. The left-hand side contains the conservative terms: namely, $D=+1(-1)$, which is for the anomalous (normal) dispersion propagation regime, and $\nu$, which represents, if negative, the saturation coefficient of the Kerr nonlinearity. In the following, the dispersion is anomalous and $\nu$ is kept relatively small. The right-hand side of Eq. (1) includes all dissipative terms: $\delta, \epsilon, \beta$, and $\mu$ are the coefficients for linear loss (if negative), nonlinear gain (if positive), spectral filtering (if positive), and saturation of the nonlinear gain (if negative), respectively. This distributed equation finds application in modeling, for instance, a wideaperture active optical cavity in the regime of short pulse operation. The model includes the effects of two-dimensional transverse diffraction of the beam and longitudinal dispersion of the pulse and its evolution along the cavity. Dissipative terms describe gain and loss of the pulse in the cavity. Higher-order dissipative terms are responsible for the nonlinear transmission characteristics of the cavity, which allows, for example, passive mode locking. This equation is a natural extension of the one-dimensional CGLE.

\section{B. General collective variable approach}

The dynamics of a light bullet is completely specified by the solution of Eq. (1) — say, $\psi(x, y, t, z)$ - but this solution describes not only a collective entity (localized in time and space), but also all other localized or nonlocalized excitations, such as noise, or radiation, that may be more or less present in the real system. Moreover, the solution may not only be able to translate as a whole entity, but it may also execute internal vibrations. In this context, it is useful, then, to somehow simplify its characterization by using a lowdimensional equivalent mechanical system based on a finite number of degrees of freedom [24]. Each degree of freedom can then be described by means of a coordinate called collective variable $(\mathrm{CV})$. The general idea in the $\mathrm{CV}$ approach (CVA) is to associate CVs with the solution's parameters of interest for which equations of motion may be derived. One may introduce $N$ collective variables, $z$ dependent-say, $X_{i}$, $i=1, \ldots, N$-in such a way that each of them can correctly describe a fundamental parameter of the solution. To this end, one can decompose the field $\psi(x, y, t, z)$ in the following way $[22,24]$ :

$$
\psi(x, y, t, z)=f\left(X_{1}, \ldots, X_{N}, x, y, t\right)+q(x, y, t, z),
$$

where the trial function $f$ is a function of the CVs and $q(x, y, t, z)$ is a residual field that represents all other excita- 
tions in the system (noise, radiation, dressing field, etc.). The precise form of the trial function which introduces the CVs in the theory is rather crucial, especially when approximations are made. After choosing the trial function one can pursue the process of characterization of the pulse in two completely different ways depending on the level of accuracy desired. First, one can make use of the exact pulse field to obtain the pulse parameters. In other words, one can take the residual field into account and carry out a numerical procedure to determine the $\mathrm{CVs}$ through a direct minimization of the residual-field energy [22]. This approach leads generally to results which are more accurate than those of the variational approach, but its application to the characterization of the propagation of light bullets would be too time consuming. The second approach of characterization of light bullets would be to carry out a variational analysis neglecting the residual field. The approximation of neglecting the residual field is called the bare approximation [22]. The first major advantage of the variational analysis lies in the fact that the pulse propagation can be completely characterized without having to know the exact pulse field $\psi(x, y, t, z)$ - that is, without having to solve the CGLE. Consequently, the amount of calculations required by the variational analysis represents only an extremely small fraction of the calculations required by any method that uses the exact pulse field throughout pulse propagation. The second major advantage of the variational analysis is that it gives a detailed qualitative picture of the role and mode of action of each term of the CGLE. The only drawback of the variational method lies in its level of accuracy, which depends very strongly on the choice of the trial function. Consequently, when approximations are made, such as the use of the variational approach, the precise shape of the trial function that introduces the CVs in the analysis of the dynamics of light bullets becomes rather crucial. At some stage, the predictions obtained by the CVA in the bare approximation should be validated by an exact solution of the CGLE in a limited set of cases.

\section{Choice of the trial function}

The choice of the trial function is important for the success of the technique. There is no known general analytical expression of the solution of the CGLE. Therefore, we do not have a perception of the spatiotemporal profile of the solution in a given parameter region. In other words, it is impossible to get any idea of the exact profile without solving numerically the CGLE [Eq. (1)]. Hence, the choice of a particular trial function has a certain degree of arbitrariness, stressing furthermore the approximate nature of the CV approach.

Thus, for systems as complex as those governed by the CGLE, it is preferable to carry out the study with at least two trial functions that have clearly different spatiotemporal profiles. Assembling together plausible stability domains predicted by various trial functions must, in principle, give a better prediction than the one obtained from a single arbitrarily chosen ansatz, before an exact numerical solving of the CGLE can be made for comparison. The simplicity of the approach makes it quick in execution, thus allowing us to carry out parallel studies with several trial functions of different profiles in vast domains of the CGLE parameters. We noticed important differences in their respective predictions. The functions that we considered are the following.

(i) The Gaussian trial function is given by

$$
f_{G 0}=X_{1} \exp \left(-\frac{t^{2}}{X_{2}^{2}}-\frac{r^{2}}{X_{3}^{2}}+i X_{4} t^{2}+i X_{5} r^{2}+i X_{6}\right),
$$

where $X_{i=1, \ldots, 6}$ represent the collective variables: $X_{1}$ stands for the pulse amplitude, $X_{2}$ and $X_{3}$ are related to the temporal and transverse widths, and $X_{4}$ and $X_{5}$ are the temporal and transverse chirp parameters. $X_{6}$ is the global phase that evolves along with propagation: when a stationary regime is reached, the phase becomes a linear function of the propagation distance $z$. There is no complete symmetry between the temporal and spatial variables, due to the existence of spectral filtering in the temporal domain. Consequently, the temporal and spatial variables are associated with different collective variables in the trial function. We also emphasize that since the system is dissipative, the solutions acquire both spatial and temporal phase chirps. The main advantage of the Gaussian trial function lies in its simplicity, which makes the procedure of derivation of the variational equations relatively easy. The drawback lies in the fact that it excludes such behaviors of internal dynamics as the steepening of the fronts of the pulse, the flattening of its crest, or the creation of an asymmetry in its temporal or spatial profiles.

(ii) To see the impact of the shape of the trial function, one can use a super-Gaussian function, which possesses steeper fronts than the Gaussian, such as

$$
f_{S G}=X_{1} \exp \left(-\frac{t^{2}}{X_{2}^{2}}-\frac{t^{4}}{X_{2}^{4}}-\frac{r^{2}}{X_{3}^{2}}-\frac{r^{4}}{X_{3}^{4}}+i X_{4} t^{2}+i X_{5} r^{2}+i X_{6}\right) \text {. }
$$

The Gaussian and super-Gaussian trial functions introduced above assume the rotational symmetry of the pulse in the transverse plane $(x, y)$. This allows us to use the transverse coordinate $r=\sqrt{x^{2}+y^{2}}$ in their analytical expressions. In other words, both trial functions do not allow one to describe symmetry breaking in the transverse plane. The latter cannot be excluded in the case of pulse propagation governed by the CGLE. To take into account this type of behavior, it is necessary to change the trial function as specified below.

(iii) To describe a richer variety of dynamical behaviors, one can use a Gaussian trial function which admits asymmetric pulse shapes in the transverse plane $(x, y)$ and is given by

$$
\begin{aligned}
f_{G 1}= & X_{1} \exp \left(-\frac{t^{2}}{X_{2}^{2}}-\frac{x^{2}}{X_{3}^{2}}-\frac{y^{2}}{X_{4}^{2}}\right. \\
& \left.+i \frac{1}{2} X_{5} t^{2}+i \frac{1}{2} X_{6} x^{2}+i \frac{1}{2} X_{7} y^{2}+i X_{8}\right) .
\end{aligned}
$$

Here $X_{3}$ and $X_{4}$ are related to the spatial widths of the pulse along the $x$ and $y$ directions, respectively.

(iv) A Gaussian trial function which can describe more elaborate asymmetric deformations of the pulse in the transverse plane $(x, y)$ is given by 


$$
\begin{aligned}
f_{G 2}= & X_{1} \exp \left(-\frac{t^{2}}{X_{2}^{2}}-\frac{x^{2}}{X_{3}^{2}}-\frac{y^{2}}{X_{4}^{2}}-X_{5} x y+i \frac{1}{2} X_{6} t^{2}+i \frac{1}{2} X_{7} x^{2}\right. \\
& \left.+i \frac{1}{2} X_{8} y^{2}+i \frac{1}{2} X_{9} x y+i X_{10}\right)
\end{aligned}
$$

Additional collective variables release new degrees of freedom that can unveil more complex dynamics, such as asymmetric pulsations. Obviously, there is a reasonable limit to the number of collective variables in order to maintain the mathematical tractability of the variational equations.

\section{Application of the CVA}

\section{Variational equations}

Hence, to proceed quite far in the analytical analysis one can make use of the lowest-order approximation of the CVA (bare approximation), which is obtained by setting the residual field to zero $[q(x, y, t, z)=0]$ in Eq. (2). Then, applying the bare approximation to the CGLE-that is, substituting $\psi=f$ in Eq. (1) (where $f$ designates a given trial function) and projecting the resulting equations in the direction of $\frac{\partial f^{*}}{\partial X_{k}}$, $k=1, \ldots, N$-yields the variational equations [22]. In the case of the simplest Gaussian function $f=f_{G 0}$, the six collective variables evolve according to the following set of six coupled ODEs:

$$
\begin{gathered}
\dot{X}_{1}=X_{1} \delta+\frac{3}{4} X_{1}^{3} \epsilon-\frac{2 \beta X_{1}}{X_{2}^{2}}+\frac{5}{9} X_{1}^{5} \mu-X_{1} X_{4} D-4 X_{1} X_{5}, \\
\dot{X}_{2}=2 X_{2} X_{4} D-\frac{1}{4} X_{2} X_{1}^{2} \epsilon-\frac{2}{9} X_{1}^{4} X_{2} \mu+\left(1-X_{2}^{4} X_{4}^{2}\right) \frac{2 \beta}{X_{2}}, \\
\dot{X}_{3}=4 X_{3} X_{5}-\frac{1}{4} X_{3} X_{1}^{2} \epsilon-\frac{2}{9} X_{1}^{4} X_{3} \mu \\
\dot{X}_{4}=-\frac{X_{1}^{2}}{2 X_{2}^{2}}+\left(\frac{1}{X_{2}^{4}}-X_{4}^{2}\right) 2 D-\frac{4 \nu X_{1}^{4}}{9 X_{2}^{2}}-\frac{8 \beta X_{4}}{X_{2}^{2}}, \\
\dot{X}_{5}=-\frac{X_{1}^{2}}{2 X_{3}^{2}}+\frac{4}{X_{3}^{4}}-4 X_{5}^{2}-\frac{4 \nu X_{1}^{4}}{9 X_{3}^{2}} \\
\dot{X}_{6}=2 \beta X_{4}+\frac{3 X_{1}^{2}}{4}-\frac{D}{X_{2}^{2}}-\frac{4}{X_{3}^{2}}+\frac{5 \nu X_{1}^{4}}{9} .
\end{gathered}
$$

For the three other trial functions under consideration, the corresponding variational equations are given in the Appendix.

\section{Fixed points and their stability}

A major goal of our study is to provide a quick approximate mapping of the regions of existence of stable and unstable solutions in the parameter space of the $(3+1)$ D CGLE. The fixed points (FPs) of the system are found by imposing the left-hand side of Eqs. (7) to be zero-i.e., $\dot{X}_{i}=0$ ( $i$ $=1, \ldots, N)$. The threshold of existence of FPs can be esti- mated by the relation $\epsilon_{s} \approx 2 \sqrt{\delta \mu}$ [12]. If $\epsilon>\epsilon_{s}$, we have in general both stable and unstable fixed points. The stability of the FPs is determined by the analysis of the eigenvalues $\lambda_{j}$ (with $j=1, \ldots, N$ ) of the Jacobian matrix $M_{i j}=\partial \dot{X}_{i} / \partial X_{j}$. The stability criterion is the following: if the real part of at least one of the eigenvalues is positive, then the corresponding FP is unstable. Thus, to have a stable FP, the real parts of the eigenvalues of the matrix $M_{i j}$ have to be all negative [8]. The stable fixed points correspond to stable solutions of the CGLE [Eq. (1)].

By investigating the parameter regions situated in the neighborhood of $(D=1, \beta=0.1, \delta=-0.4, \mu=-0.1)$, we find in the plane $(\nu, \epsilon)$ a rich variety of solutions of Eq. (1), including stationary spatiotemporal solitons-i.e., dissipative light bullets (LBs). In practice, the set of equations $\dot{X}_{i}=0$ is solved by means of a fourth-order Runge-Kutta algorithm by providing an initial condition-for instance, $\psi(x, y, t, 0)$ $=4 \exp \left(-\frac{t^{2}}{1.3}-\frac{x^{2}}{1}-\frac{y^{2}}{0.9}\right)$-for a given set of CGLE parameters. When a stable fixed point is found-say, $X_{i 0}$ - it serves as the initial condition for solving $\dot{X}_{i}=0$ for the neighbor CGLE parameters.

\section{STATIONARY LIGHT BULLETS}

\section{A. Application of the variational approach to the determination of stable LBs}

As mentioned above, a variational approach is an approximate method with a level of accuracy that depends strongly on the particular trial function that is chosen. Consequently, some care must be taken in its application to an elaborate equation such as the CGLE. It is useful then, at the preliminary stage of such a study, to have clear insight into the correctness of the CVA, at least in a small portion of the whole parameter space under consideration. This can be done through a direct comparison between the results given by the CVA with those given by the entirely numerical procedure. To this end, we started the search for stationary LBs by using the CVA in a domain of parameters where LBs were found recently from the exact numerical solution of Eq. (1) $[5,25]$. We fixed the values of four parameters of Eq. (1) namely, $D=1, \delta=-0.4, \beta=0.1$, and $\mu=-0.1$-and varied the two remaining parameters. Thus, we have explored the $(\epsilon, \nu)$ plane, and we have observed the following features.

(i) The CVA using the Gaussian trial function $f_{G 0}$ predicts the stability domain indicated in dark gray color in Fig. 1. One can clearly observe that most of the predicted domain falls inside the exact stability domain (which lies between the dashed lines in Fig. 1), but occupies only a small fraction of the whole stability domain. In fact, the Gaussian trial function allows only the prediction of the stability domains in which the exact profile is not too far from the Gaussian shape. More generally, one can hardly predict the totality of a large stability domain by means of a single trial function, because a large domain includes necessarily quite different profiles.

(ii) The CVA using the super-Gaussian trial function $f_{S G}$ predicts the stability domain which appears in Fig. 1 as a hatched domain. This domain contains entirely the one pre- 


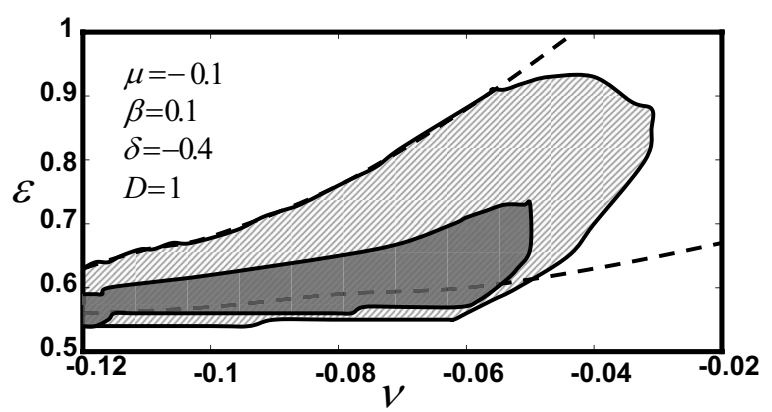

FIG. 1. Map of stable LBs found from the CVA in the $(\nu, \epsilon)$ plane, using either a Gaussian (domain in dark gray) or a superGaussian trial function (hatched domain). Other CGLE parameters appear in the figure. For comparison, the domain found with exact numerical solution of the CGLE lies inside the two dashed lines.

dicted by the Gaussian trial function and expands over a much larger part of the whole stability region found by exact calculations. Thus, Fig. 1 shows that the CVA with use of the super-Gaussian trial function provides an approximate but fairly accurate prediction of the stability domain of stationary light bullets. It is worth noting that further improvements may be achieved by using more elaborate trial functions, but here, we do not intend to go further in this direction.

It is interesting to gain insight from a simple and useful quantity, which is the total pulse energy, defined as

$$
Q(z)=\int_{-\infty}^{+\infty}|\psi(x, y, t, z)|^{2} d x d y d t
$$

Figure 2 represents the energies obtained with the Gaussian (triangles) and the super-Gaussian trial functions (black line) compared to the energy of the exact solution (circles) when the parameter $\nu$ is varied and $\epsilon$ is fixed to 0.6. One can clearly see that the energy of the super-Gaussian coincides rather well with the energy of the exact soliton solution. This seems an indication that the profile of the exact soliton solution should be relatively close to a super-Gaussian shape for the related parameters $(\epsilon, \nu)$. However, the comparison of profiles in the case of $(3+1) \mathrm{D}$ spatiotemporal solitons is not

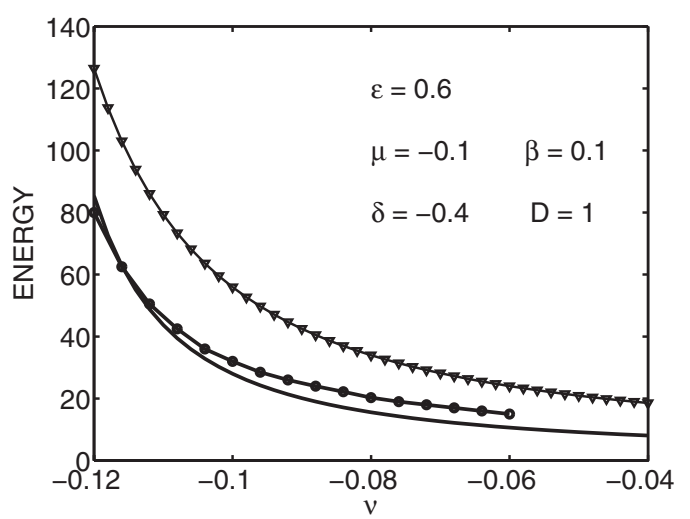

FIG. 2. Energy $Q$ versus parameter $\nu$ obtained with three different methods: using the CVA with the Gaussian (triangles), superGaussian (black line) trial functions, and direct numerical simulations of the CGLE (circles).
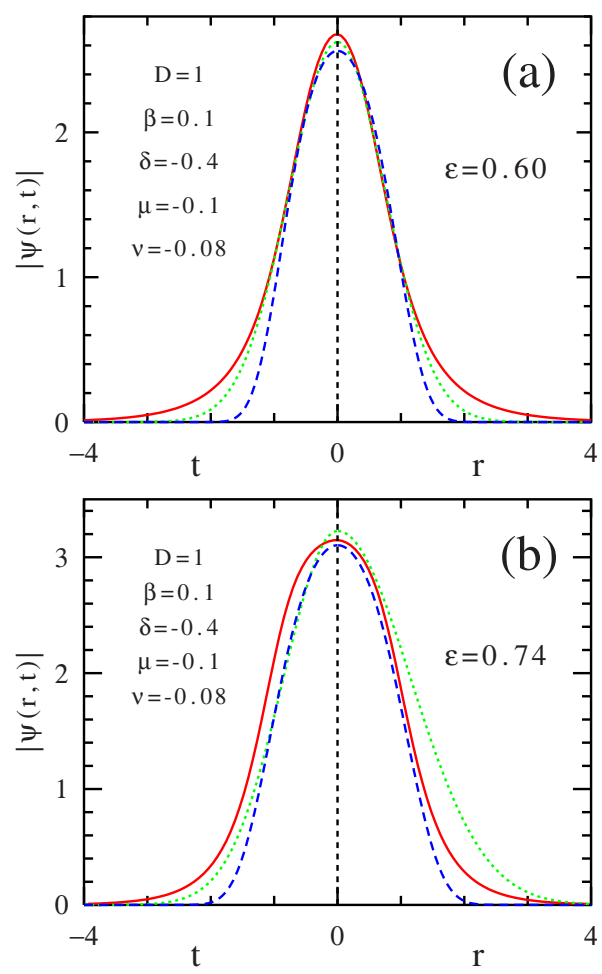

FIG. 3. (Color online) Comparison of transverse $(r)$ and temporal $(t)$ profiles of 3D light bullets obtained through exact calculations (solid red line), CVA with the Gaussian trial function (dotted green line), and CVA with the super-Gaussian trial function (dashed blue line), for the following cases: (a) $\epsilon=0.60$ and (b) $\epsilon=0.74$, the rest of the parameters being identical.

as straightforward, as discussed below. Finally, it is worth noting that the self-stabilization of a light bullet requires some minimal amount of energy when the gain and loss parameters are fixed. So, if the energy of the solution drops along with the increase of $\nu$, which is a dispersive parameter, there is a value of $\nu$ at given $\epsilon$ where the solution becomes unstable and the field vanishes. Other stable solutions can then be found at the expense of an increase of the nonlinear gain parameter, hence the shape of the domains in Fig. 1.

The comparison of the above three soliton solution profiles is presented in Fig. 3 for two different values of $\epsilon$, the rest of the parameters being identical. When $\epsilon=0.60$ [Fig. $3(\mathrm{a})]$, it is in fact the Gaussian profile which is the closest to the profile of the exact solution. This can be understood from Fig. 1, since the parameters coincide with the central part of the domain of stable LB with the Gaussian trial function. Note also that both temporal and spatial profiles are very similar. The situation changes completely when $\epsilon$ moves to the value 0.74 . The spatial profile of the super-Gaussian becomes the closest. Indeed, the Gaussian solution is no longer stable for this value of $\epsilon$. For the parameters in the latter case, the spherical symmetry of solutions is also broken, so that temporal and transverse profiles differ.

\section{B. Predictions of the existence of LBs in other parameter planes}

Comparing with the exact numerical calculations, which take from hours to even days for a single set of parameters, 

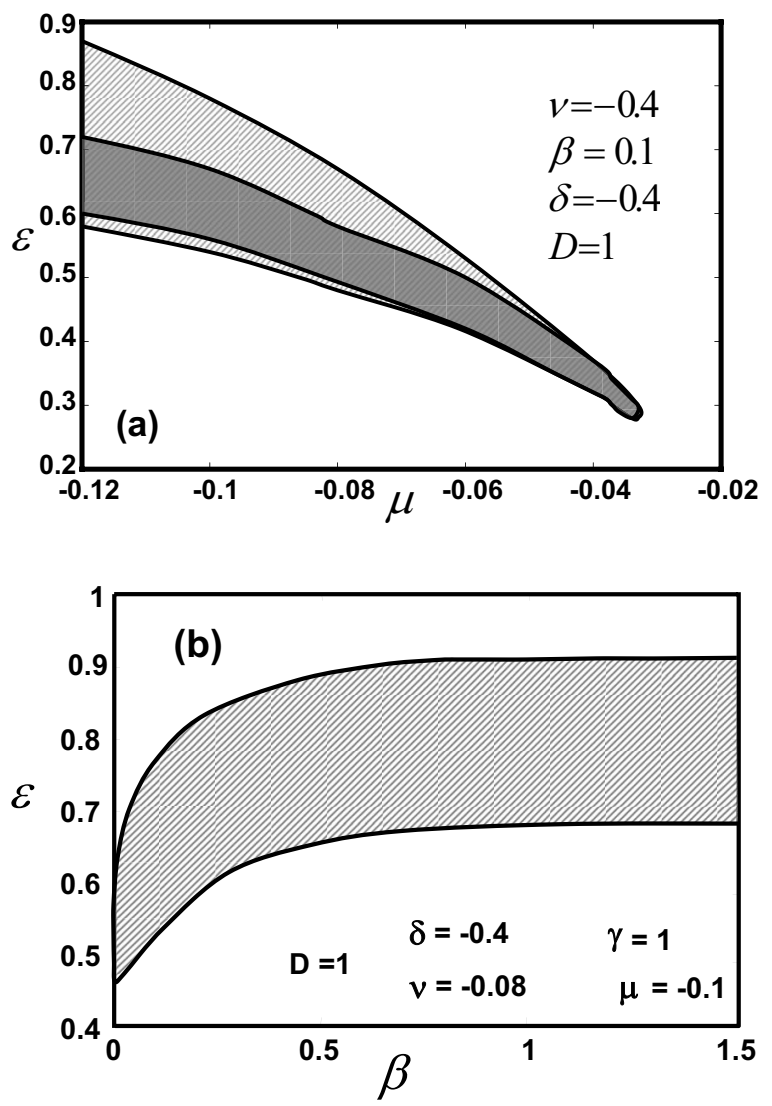

FIG. 4. Domains of stationary LBs found from CVA in (a) the $(\mu, \epsilon)$ plane and (b) the $(\beta, \epsilon)$ plane. In (a), the domain obtained with the super-Gaussian function (hatched) includes that obtained with the Gaussian function (gray). In (b), the domain is obtained with the super-Gaussian function. With the Gaussian, it was quite similar except for a part of it where the boundaries could not be found precisely.

the advantage of the CVA lies in the extremely fast solution of the ODEs (7) and of the Jacobian stability matrix. The calculation of 1000 parameter points can be performed in typically 5 min on a standard PC.

Hence, we have explored other parameter domains for which exact solutions have not been calculated yet. Two examples of maps are given in Fig. 4. Figure 4(a) presents a domain of existence for stable stationary LBs in the $(\mu, \epsilon)$ parameter plane. The shape of the existence domain is similar to the shape of the domain where stable temporal pulses were found numerically in the $(1+1) \mathrm{D}$ CGLE [8]. Although the $(3+1) \mathrm{D}$ evolution problem considered in the present paper is much more complex, we can see that the trend which links the equation parameters and the existence of dissipative solitons is similar. This seems to be a general feature for dissipative solitons, which could accommodate more easily for higher spatiotemporal dimensionality than Hamiltonian solitons. Figure 4(b) presents a domain for stable LBs in the $(\beta, \epsilon)$ plane. This domain is similar to the domain found numerically in [14] for a slightly different value of $\nu$.

The shape of these domains can be explained as follows. The quintic dissipative term of the CGLE is essential to ensure the stabilization of the stationary solutions, as was proven in the purely temporal case [8]. Its importance is controlled by the coefficient $\mu$, which should be negative. In the spatiotemporal case, a minimal value of $\mu$, which is close to -0.025 in Fig. 4(a), is thus required to obtain stable light bullets and depends on the other parameters. It represents the edge of the domain of stable LBs, hence its shape of a tip. When the magnitude of the coefficient $\mu$ becomes larger, the stabilization of LBs is improved, so that their domain of existence (with respect to a variation of the nonlinear gain coefficient $\epsilon$ ) increases, but at the same time the values of $\epsilon$ tend to increase, since the nonlinear gain needs to increase in order to compensate for the additional high-order gain saturation. We can understand the impact of the spectral filtering term, proportional to the coefficient $\beta$, on a similar basis, although the term acting in the CGLE is linear. Indeed, spectral filtering acts as a localizing process in the spectral domain, which in turn stabilizes the temporal pulse profile. But it also represent extra loss that can be compensated for by an increase of the nonlinear gain coefficient $\epsilon$, hence the shape of Fig. 4(b).

We see that the principle of balance between dissipative linear and nonlinear terms provides relatively wide domains for the existence of stable light bullets. We are now interested in the type of localized solutions that lie close to the boundaries where stable LBs have been found. This is discussed in the next section.

\section{PULSATING LIGHT BULLETS}

In the two-dimensional plane of parameters, the regions of existence of stable stationary LBs presented above are contained between an upper and a lower values of $\epsilon$. Below the lower limit, the energy pumped into the system is not enough to support the LBs. In this case the localized solution dissipates and eventually vanishes. On the contrary, when $\epsilon$ is above the upper value, the energy supply inflates the LB to the extent that it grows indefinitely in size. However, this process does not occur in one single step. There is a small intermediate region of $\epsilon$ values where stationary solutions are transformed into pulsating ones before the continuous inflating begins at even higher $\epsilon$.

\section{A. Radially symmetric pulsations in the $(x, y)$ plane}

Exploring the dynamics of collective variables for fixed points located above the upper boundary of $\epsilon$, we found pulsating solutions in the form of stable limit cycles. These solutions can be considered as attractors. The dynamics is illustrated in Fig. 5. Figure 5(a) shows the evolution of the total energy of the $\mathrm{LB}$, starting from an initial value $Q \simeq 55$ of the trial function $f_{S G}$. The solution is unstable, and after the onset of oscillations, the pulsating dynamics becomes steady. It is characterized by large energy oscillations between the two constant limits. The pulse breathing in the transverse and in the temporal domain can be seen from the evolution plots of Figs. 5(b) and 5(c).

The domain of existence for such pulsations is relatively large, as displayed in Fig. 6. This is the signature of the existence of relatively strong attractors of the limit-cycle type. Although this remarkable feature has been observed in 

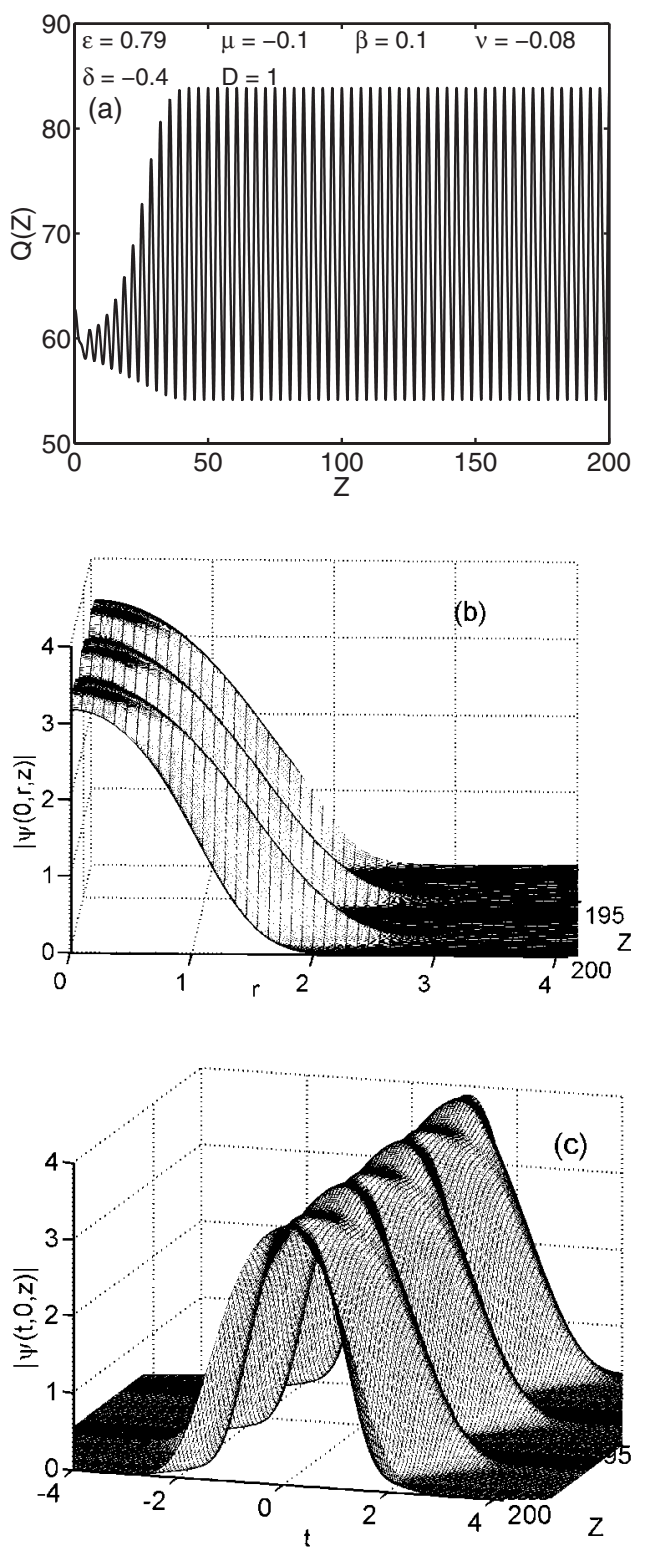

FIG. 5. Pulsations of a light bullet reconstructed from the collective variable approach. (a) Evolution of the total pulse energy, showing the onset of pulsations. Solution of the CVA dynamical system is converged to a stable limit cycle at $z=50$. (b) Evolution of the radial field profile. Pulsations of this LB are radially symmetric. (c) Evolution of the temporal field profile.

a variety of nonlinear dissipative optical systems $[17,21,25-28]$, we have to bear in mind that the existence of such dynamics needs confirmation by the direct numerical simulations of the original CGLE in the neighborhood of the parameters used for the CVA. The role of the CVA is indeed the fast exploration of the dynamics in parameter domains. But as we have seen previously, the type of the trial function is crucial in getting close to numerical results. Obviously, the type of pulsation itself is linked to the choice of the trial function and the number of collective variables. With a trial function possessing rotational symmetry in the transverse $(x, y)$ plane, such as $f_{G 0}$ and $f_{S G}$, we can only expect breathing-type pulsations, whereas the full dynamics of the

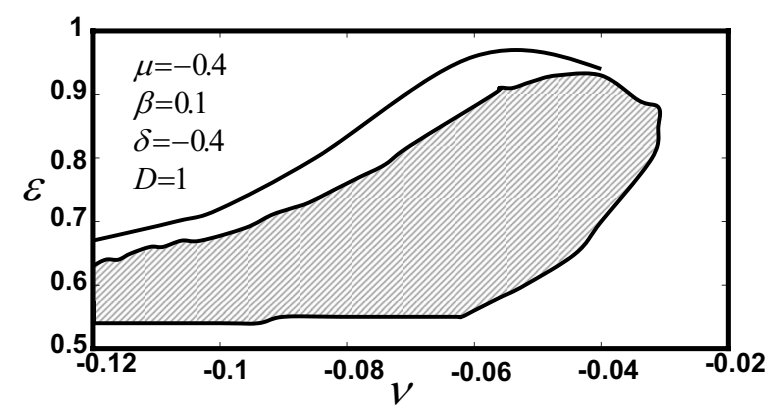

FIG. 6. Regions of existence for pulsating and stationary LBs. Stable pulsating LBs (white area) are located just above the region of stable stationary ("plain") LBs (light gray area). These results are obtained using the CVA and the super-Gaussian trial function.

CGLE solutions can be much more complicated.

In the present case, the finding of radially symmetric breathing-type pulsations with the help of CVA is confirmed by direct numerical calculations of the CGLE as well. These numerical results are presented in Fig. 7. We can see the steady pulsations over relatively large interval of propagation distances (from $z=50$ to 140 ). However, the simulations also show that the breathing-type pulsating dynamics is unstable. After $z=140$, this instability triggers the formation of a rotating double-bullet complex [25]. The switching between the two types of dynamics is clearly illustrated by the snapshots of the evolution in the transverse $(x, y)$ plane in Fig. 8. Plots at $z=112.9$ and $z=115.7$ show the two opposite phases of the radially symmetric breathing dynamics, whereas the plot at $z=156$ shows the appearance of the double-bullet complex.

\section{B. Symmetry breaking of pulsations}

In order to study more complicated pulsating dynamics, we used a more elaborate Gaussian trial function that can break the transverse cylindrical symmetry. Starting from $f_{S G}$ which has six collective variables, we moved to $f_{G 1}$ and $f_{G 2}$, which have eight and ten collective variables, respectively. The ODEs governing the evolution of the collective variables in each case are provided in the Appendix.

\section{Asymmetric pulsations}

The trial function $f_{G 1}$ allows us to model an asymmetric evolution of the field profiles along the $x$ and $y$ axes. An example of such dynamics is presented in Fig. 9 for the following values of the parameters: $\epsilon=0.63, \nu=-0.08, \delta=$ -0.4 , and $\beta=0.1$. Small oscillations of the LBs appear right after starting the evolution from a certain symmetric localized field as initial condition. They gradually develop in amplitude (a), temporal width (b), and spatial widths (c), (d). These oscillations become stationary at $z>700$, and the close-up view of oscillations (e)-(h) shows a nearly harmonic evolution of the collective variables. We can notice that the $x$ and $y$ oscillations are out of phase and have the same amplitude. This shows periodic out of phase consecutive contractions of the LB in the $x$ and $y$ directions. At the same time, the total energy is not a constant and the ampli- 

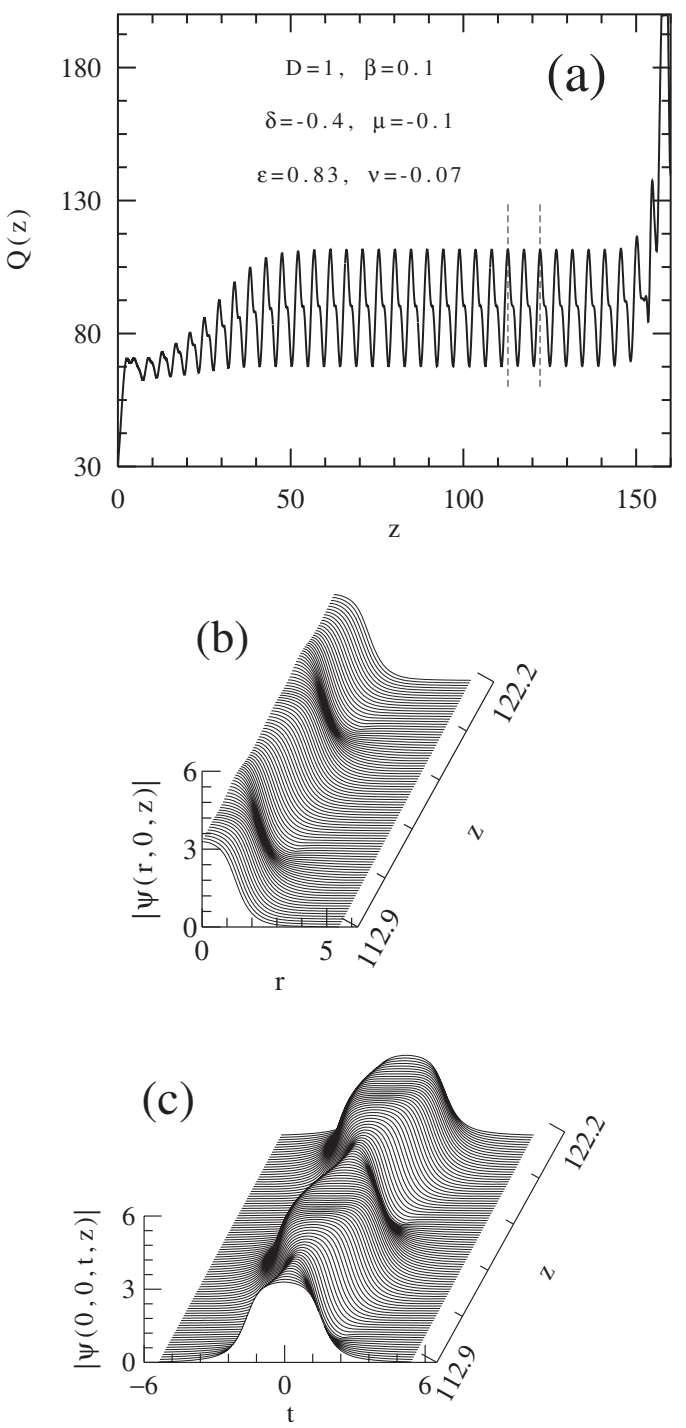

FIG. 7. Radially symmetric pulsations of a LB found with the direct numerical computations of the CGLE in the vicinity of the parameters used in Fig. 5. (a) Evolution of energy. The plot shows the convergence to steady pulsations and subsequent instability at $z \approx 150$. (b) Evolution of the transverse intensity profile and (c) evolution of the temporal intensity profile over two pulsation periods located between the two dashed vertical lines in (a).

tude and temporal width oscillate at a doubled frequency.

A slight increase of the nonlinear gain parameter results in an increase of the oscillation amplitudes, which become anharmonic. This is illustrated in Fig. 10, plotted for the value of $\epsilon=0.66$. The onset of pulsations occurs quicker, but regular oscillations appear after the initial amplitude overflowing effect. The pulsations of LBs along the $x$ and $y$ axes acquire a difference in amplitude and shape. We verified that these results are not numerical artifacts. In a succession of simulations, in about half of the simulated cases, we observed the swapping of the roles of $x$ and $y$ in pulsation dynamics. This is one of the features of the symmetry-breaking phenomenon. However, such pulsations have not yet been found in direct numerical simulations of the CGLE, where symmetry breaking is often associated with the onset of a rotational
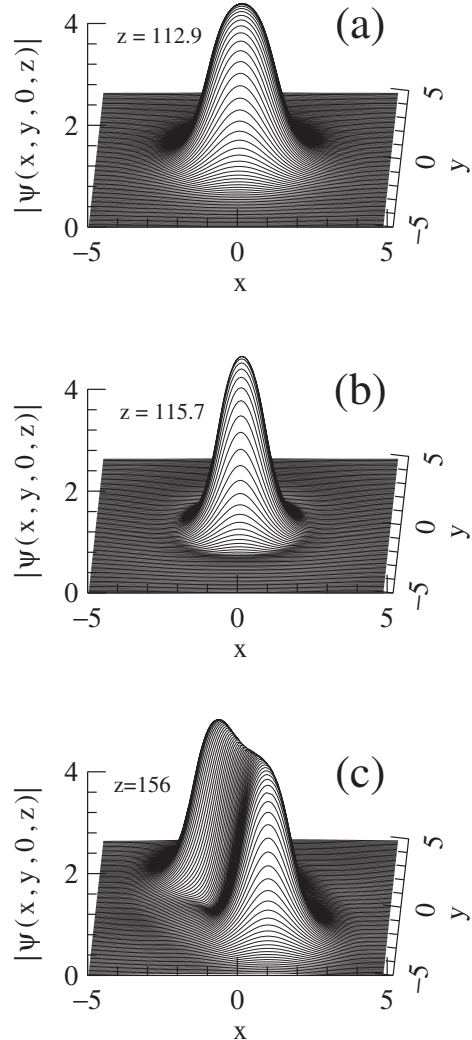

FIG. 8. Spatial profiles of the light bullets obtained from direct numerical simulations of the original PDE at three consecutive propagation distances: (a) $z=112.9$, (b) $z=115.7$, and (c) $z=156$, at $t=0$. For the values of $z$ between 50 and 150 , pulsations are radially symmetric in accordance with the results predicted by the CVA. However, at $z=150$, the radial symmetry breaking results in a rotating double-bullet complex. The corresponding transverse profile at $t=0$ is shown in (c).

motion, as discussed in Sec. IV A. It is clear that rotation is a frozen degree of freedom when the trial function $f_{G 1}$ is used; thus, we tried to include rotations using the trial function $f_{G 2}$.

\section{Rotating $\mathbf{L B S}$}

In the region of equation parameters where pulsations can be triggered easily, we were able to start a rotational motion using the trial function $f_{G 2}$. These were initiated through breaking of radial symmetry. Both clockwise and counterclockwise motions of the transverse field profile can be observed in propagation along the $z$ axis. However, we were not able to observe stable pulsations using collective variables. The field expands in transverse directions with the total energy increasing indefinitely. We believe that there are two main reasons for the limitations of the CVA. First, increasing the dimensionality of the dynamical system governing the evolution reduces the chances of calculating accurately the domains of existence of stable LBs. Second, exact numerical simulations show that near the domain of existence of stable LBs pulsations have several periods. We observed a variety of rotating double-bullet complexes which can be considered as two and more periodic stable pulsating 

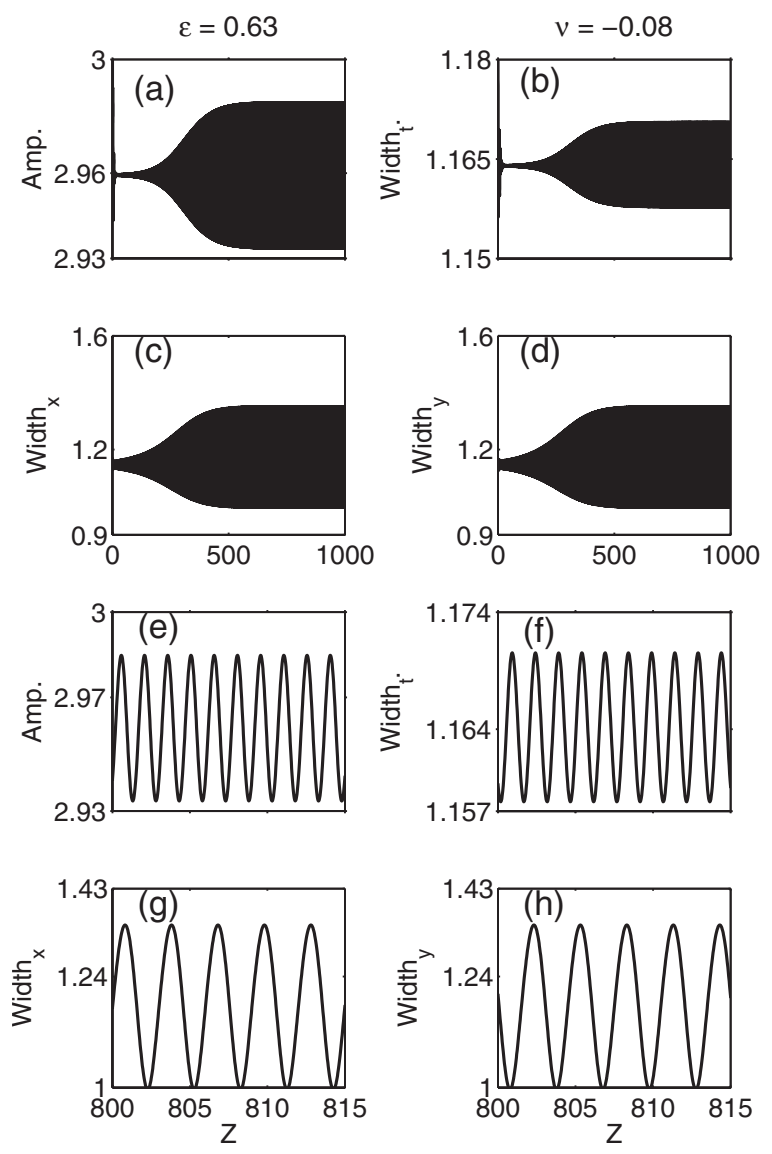

FIG. 9. Radially asymmetric pulsations obtained with the use of the CVA and the trial function $f_{G 1}$. The evolution of the following collective variables with respect to $z$ is shown: (a) the amplitude $X_{1}$, (b) the temporal width $X_{2}$, (c) the width along the $x$ axis, $X_{3}$, and (d) the width along the $y$ axis, $X_{4}$. The onset of stable harmonic pulsations appears clearly. Enlarged views of those pulsations are plotted in (e)-(h). The pulsations along the $x$ and $y$ axes are out of phase and share the same amplitude.

solutions. For example, the exact profile shown in Fig. 8(c) cannot be represented as a simple "rotating" Gaussian function $f_{G 2}$. Consequently, an attempt to describe rotating double-bullet complexes by increasing the number of collective variables in the CVA fails. Using more variables in the trial function, we lose the simplicity and tractability of this approach.

\section{CONCLUSIONS}

In the present paper, we have demonstrated that the collective variable approach is a useful tool for predicting approximately the domains of existence of stable light bullets in the parameter space of the $(3+1) \mathrm{D}$ complex cubic-quintic Ginzburg-Landau equation. It is very efficient for approximating stationary soliton solutions when a suitable trial function is chosen. We could even predict pulsating solutions of a breathing type using the CVA. Altogether, prediction results are in a fairly good agreement with the direct numerical solutions of the CGLE, qualitatively as well as quantitatively, and this can hold for relatively large domains of the CGLE parameters.
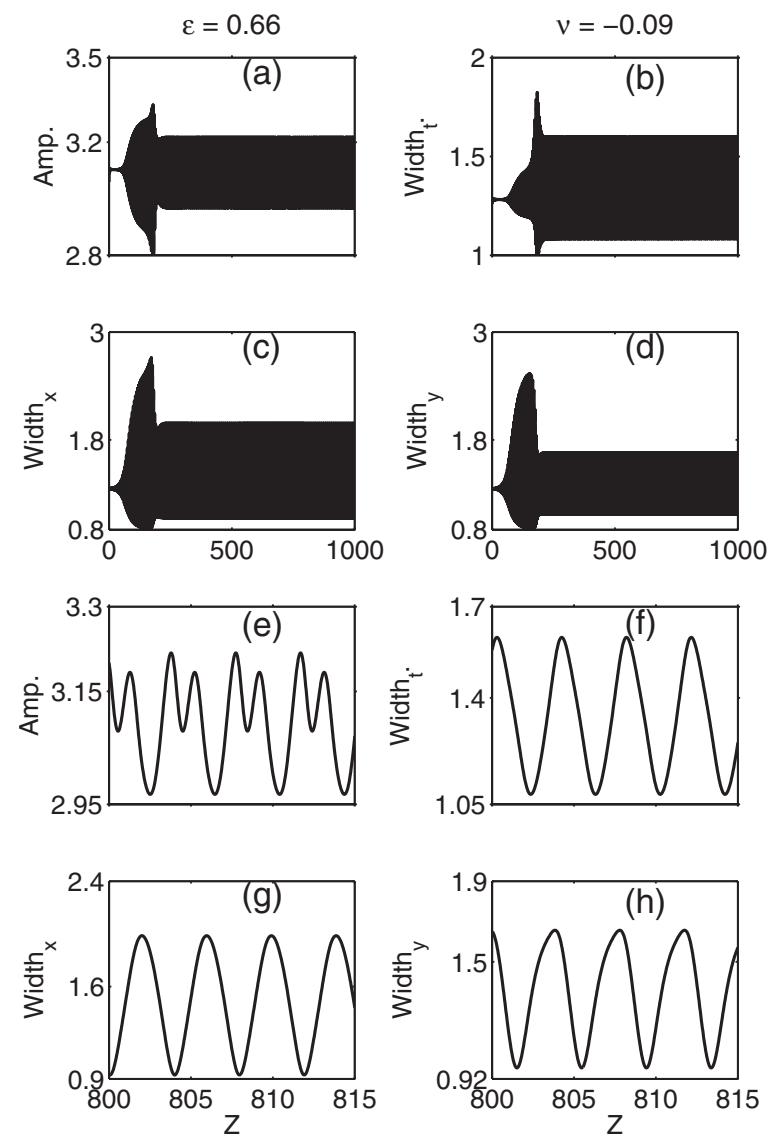

FIG. 10. Quasiperiodic (multifrequency) radially asymmetric pulsations produced when we increased the nonlinear gain coefficient to $\epsilon=0.66$. Anharmonic pulsations along $x$ and $y$ are characterized by different amplitudes and shapes. Due to the symmetry of the original CGLE, pulsations along the $x$ and $y$ directions can be interchanged.

The technique is incomparably quicker than direct numerical computations. Of course, the latter should be used at the final stage of studies to confirm, complement, or invalidate the CVA predictions. The present methodology should soften the severe constraints of theoretical investigation of the dynamical behavior of light bullets in physical systems of high dimension.

\section{ACKNOWLEDGMENTS}

A.K., Ph.G., and P.T.D. were supported by Agence Nationale de la Recherche (Project No. ANR-05-BLAN-0152-01), J.M.S.C. acknowledges support from the M.E.y C. under Contract No. FIS2006-03376, and N.A. acknowledges support from the Australian Research Council's Discovery Projects funding scheme (Project No. DP0663216).

\section{APPENDIX: ODES FOR THE EVOLUTION OF THE COLLECTIVE VARIABLES FOR SEVERAL TRIAL FUNCTIONS}

For the super-Gaussian $f_{S G}$, we have 


$$
\begin{aligned}
& \dot{X}_{1}=X_{1} \delta+0.46 X_{1}^{3} \epsilon e^{1 / 2}+0.21 \mu X_{1}^{5} e-X_{1} X_{4} D-2 X_{1} X_{5} \\
& -\frac{X_{1} \beta}{X_{2}^{2}}\left(3.18+0.17 X_{2}^{4} X_{4}^{2}\right) \\
& \dot{X}_{2}=2 X_{2} X_{4} D-0.12 X_{2} X_{1}^{2} \epsilon e^{1 / 2}-0.07 X_{1}^{4} X_{2} \mu e \\
& +\left(2.63-0.81 X_{2}^{4} X_{4}^{2}\right) \frac{\beta}{X_{2}}, \\
& \dot{X}_{3}=4 X_{3} X_{5}-0.12 X_{3} X_{1}^{2} \epsilon e^{1 / 2}-0.07 X_{1}^{4} X_{3} \mu e,
\end{aligned}
$$$$
\dot{X}_{4}=\left(\frac{4.90}{X_{2}^{4}}-2 X_{4}^{2}\right) D-\frac{18.31 X_{4} \beta}{X_{2}^{2}}-\frac{0.63 X_{1}^{2} e^{1 / 2}}{X_{2}^{2}}-\frac{0.38 X_{1}^{4} \nu e}{X_{2}^{2}},
$$$$
\dot{X}_{5}=-4 X_{5}^{2}+\frac{9.80}{X_{3}^{4}}-\frac{0.63 X_{1}^{2} e^{1 / 2}}{X_{3}^{2}}-\frac{0.38 X_{1}^{4} \nu e}{X_{3}^{2}},
$$$$
\dot{X}_{6}=2.65 \beta X_{4}+0.25 X_{1}^{4} \nu e+0.52 X_{1}^{2} e^{1 / 2}-\frac{3.28}{X_{3}^{2}}-\frac{1.64 D}{X_{2}^{2}} .
$$

For the case of the Gaussian $f_{G 1}$, we have

$$
\begin{aligned}
\dot{X}_{1}= & X_{1} \delta+\frac{7}{16} \sqrt{2} X_{1}^{3} \epsilon-\frac{2 \beta X_{1}}{X_{2}^{2}}+\frac{2}{9} \mu \sqrt{3} X_{1}^{5}-\frac{1}{2} X_{1} X_{5} \beta_{2}-\frac{1}{2} X_{1} X_{6} \\
& -\frac{1}{2} X_{1} X_{7}
\end{aligned}
$$

$$
\dot{X}_{2}=X_{2} X_{5} \beta_{2}-\frac{1}{8} \sqrt{2} X_{2} X_{1}^{2} \epsilon-\frac{2}{27} \mu \sqrt{3} X_{1}^{4} X_{2}+\left(4-X_{2}^{4} X_{5}^{2}\right) \frac{\beta}{2 X_{2}},
$$

$$
\begin{gathered}
\dot{X}_{3}=X_{3} X_{6}-\frac{1}{8} \sqrt{2} X_{3} X_{1}^{2} \epsilon-\frac{2}{27} \mu \sqrt{3} X_{1}^{4} X_{3}, \\
\dot{X}_{4}=X_{4} X_{7}-\frac{1}{8} \sqrt{2} X_{4} X_{1}^{2} \epsilon-\frac{2}{27} \mu \sqrt{3} X_{1}^{4} X_{4}, \\
\dot{X}_{5}=-\beta_{2} X_{5}^{2}-\frac{\sqrt{2} X_{1}^{2}}{2 X_{2}^{2}}+\frac{4 \beta_{2}}{X_{2}^{4}}-\frac{8 \sqrt{3} \nu X_{1}^{4}}{27 X_{2}^{2}}-\frac{8 \beta X_{5}}{X_{2}^{2}},
\end{gathered}
$$

$$
\dot{X}_{6}=-X_{6}^{2}-\frac{\sqrt{2} X_{1}^{2}}{2 X_{3}^{2}}+\frac{4}{X_{3}^{4}}-\frac{8 \sqrt{3} \nu X_{1}^{4}}{27 X_{3}^{2}},
$$

$$
\begin{gathered}
\dot{X}_{7}=-X_{7}^{2}-\frac{\sqrt{2} X_{1}^{2}}{2 X_{4}^{2}}+\frac{4}{X_{4}^{4}}-\frac{8 \sqrt{3} \nu X_{1}^{4}}{27 X_{4}^{2}}, \\
\dot{X}_{8}=\beta X_{5}+\frac{7 \sqrt{2} X_{1}^{2}}{16}-\frac{\beta_{2}}{X_{2}^{2}}-\frac{1}{X_{3}^{2}}-\frac{1}{X_{4}^{2}}+\frac{2 \sqrt{3} \nu X_{1}^{4}}{9} .
\end{gathered}
$$

For the case of the Gaussian $f_{G 2}$, we have

$$
\begin{aligned}
\dot{X}_{1}= & X_{1} \delta+\frac{7}{16} \sqrt{2} X_{1}^{3} \epsilon-\frac{2 \beta X_{1}}{X_{2}^{2}}+\frac{2}{9} \mu \sqrt{3} X_{1}^{5}-\frac{1}{2} X_{1} X_{6} \beta_{2}-\frac{1}{2} X_{1} X_{7} \\
& -\frac{1}{2} X_{1} X_{8},
\end{aligned}
$$

$$
\dot{X}_{2}=X_{2} X_{6} \beta_{2}-\frac{1}{8} \sqrt{2} X_{2} X_{1}^{2} \epsilon-\frac{2}{27} \mu \sqrt{3} X_{1}^{4} X_{2}+\left(4-X_{2}^{4} X_{6}^{2}\right) \frac{\beta}{2 X_{2}},
$$

$$
\dot{X}_{3}=X_{3} X_{7}-\frac{1}{8} \sqrt{2} X_{3} X_{1}^{2} \epsilon-\frac{2}{27} \mu \sqrt{3} X_{1}^{4} X_{3}+\frac{1}{4} X_{5} X_{9} X_{3}^{3},
$$

$$
\dot{X}_{4}=X_{4} X_{8}-\frac{1}{8} \sqrt{2} X_{4} X_{1}^{2} \epsilon-\frac{2}{27} \mu \sqrt{3} X_{1}^{4} X_{4}+\frac{1}{4} X_{5} X_{9} X_{4}^{3},
$$

$$
\dot{X}_{5}=-X_{5} X_{7}-X_{5} X_{8}+\frac{1}{4} \sqrt{2} \epsilon X_{1}^{2} X_{5}+\frac{4}{27} \sqrt{3} \mu X_{1}^{4} X_{5}-\frac{X_{9}}{X_{4}^{2}}-\frac{X_{9}}{X_{3}^{2}},
$$

$$
\dot{X}_{6}=-\beta_{2} X_{6}^{2}-\frac{\sqrt{2} X_{1}^{2}}{2 X_{2}^{2}}+\frac{4 \beta_{2}}{X_{2}^{4}}-\frac{8 \sqrt{3} \nu X_{1}^{4}}{27 X_{2}^{2}}-\frac{8 \beta X_{6}}{X_{2}^{2}},
$$

$$
\begin{gathered}
\dot{X}_{7}=X_{5}^{2}-X_{7}^{2}-\frac{X_{9}^{2}}{4}-\frac{\sqrt{2} X_{1}^{2}}{2 X_{3}^{2}}+\frac{4}{X_{3}^{4}}-\frac{8 \sqrt{3} \nu X_{1}^{4}}{27 X_{3}^{2}}, \quad(\text { A21 }) \\
\dot{X}_{8}=X_{5}^{2}-X_{8}^{2}-\frac{X_{9}^{2}}{4}-\frac{\sqrt{2} X_{1}^{2}}{2 X_{4}^{2}}+\frac{4}{X_{4}^{4}}-\frac{8 \sqrt{3} \nu X_{1}^{4}}{27 X_{4}^{2}}, \quad(\text { A22 }) \\
\dot{X}_{9}=-X_{9} X_{7}-X_{9} X_{8}-\frac{1}{2} \sqrt{2} X_{1}^{2} X_{5}-\frac{8}{27} \sqrt{3} \nu X_{1}^{4} X_{5}+\frac{4 X_{5}}{X_{4}^{2}}+\frac{4 X_{5}}{X_{3}^{2}},
\end{gathered}
$$

$$
\dot{X}_{10}=\beta X_{6}+\frac{7 \sqrt{2} X_{1}^{2}}{16}-\frac{\beta_{2}}{X_{2}^{2}}-\frac{1}{X_{3}^{2}}-\frac{1}{X_{4}^{2}}+\frac{2 \sqrt{3} \nu X_{1}^{4}}{9} \text {. }
$$


[1] G. Nicolis and I. Prigogine, Self Organization in Nonequilibrium Systems-From dissipative structures to order through fluctuations (Wiley, New York, 1977).

[2] N. Akhmediev and A. Ankiewicz, Dissipative Solitons (Springer, Heidelberg, 2005).

[3] N. Akhmediev and A. Ankiewicz, Dissipative Solitons: From Optics to Biology and Medicine in Springer Lecture Notes in Physics (Springer, Heidelberg, 2008), Vol. 751.

[4] N. N. Rosanov, Spatial Hysteresis and Optical Patterns (Springer, Berlin, 2002).

[5] P. Grelu, J. M. Soto-Crespo, and N. Akhmediev, Opt. Express 13, 9352 (2005).

[6] P. L. Kelley, Phys. Rev. Lett. 15, 1005 (1965).

[7] Y. Silberberg, Opt. Lett. 15, 1282 (1990).

[8] J. M. Soto-Crespo, N. Akhmediev, and V. V. Afanasjev, J. Opt. Soc. Am. B 13, 1439 (1996).

[9] I. S. Aranson and L. Kramer, Rev. Mod. Phys. 74, 99 (2002).

[10] J. M. Soto-Crespo, N. N. Akhmediev, V. V. Afanasjev, and S. Wabnitz, Phys. Rev. E 55, 4783 (1997).

[11] A. I. Maimistov, JETP 77, 727 (1993).

[12] E. N. Tsoy, A. Ankiewicz, and N. Akhmediev, Phys. Rev. E 73, 036621 (2006).

[13] N. Veretenov, A. Vladimirov, N. Kaliteevskii, N. Rosanov, S. Fedorov, and A. Shatsev, Opt. Spectrosc. 89, 380 (2000).

[14] J. M. Soto-Crespo, P. Grelu, and N. Akhmediev, Opt. Express 14, 4013 (2006).

[15] D. Mihalache, D. Mazilu, F. Lederer, Y. V. Kartashov, L. C.
Crasovan, L. Torner, and B. A. Malomed, Phys. Rev. Lett. 97, 073904 (2006).

[16] D. Mihalache, D. Mazilu, F. Lederer, H. Leblond, and B. A. Malomed, Phys. Rev. A 75, 033811 (2007).

[17] N. Akhmediev, J. M. Soto-Crespo, and P. Grelu, Chaos 17, 037112 (2007).

[18] N. Veretenov, N. Rosanov, and S. Fedorov, Opt. Quantum Electron. 40, 253 (2008).

[19] V. Skarka and N. B. Aleksić, Phys. Rev. Lett. 96, 013903 (2006).

[20] A. Ankiewicz, N. Akhmediev, and N. Devine,Opt. Fiber Technol. 13, 91 (2007).

[21] E. N. Tsoy and N. Akhmediev, Phys. Lett. A 343, 417 (2005).

[22] P. Tchofo-Dinda, A. B. Moubissi, and K. Nakkeeran, Phys. Rev. E 64, 016608 (2001).

[23] N. B. Aleksić, V. Skarka, D. V. Timotijević, and D. Gauthier, Phys. Rev. A 75, 061802 (2007).

[24] R. Boesch, P. Stancioff, and C. R. Willis, Phys. Rev. B 38, 6713 (1988).

[25] J. M. Soto-Crespo, N. Akhmediev, and P. Grelu, Phys. Rev. E 74, 046612 (2006).

[26] N. Akhmediev, J. M. Soto-Crespo, and G. Town, Phys. Rev. E 63, 056602 (2001).

[27] M. Grapinet and P. Grelu, Opt. Lett. 31, 2115 (2006).

[28] J. M. Soto-Crespo, P. Grelu, N. Akhmediev, and N. Devine, Phys. Rev. E 75, 016613 (2007). 